\title{
Relación entre el Financiamiento Fiscal, la Calidad del Cuerpo Académico y la Retención de Estudiantes Universitarios en Chile
}

\author{
Carmen A. Araneda-Guirriman ${ }^{(1)}$, Emilio R. Rodríguez-Ponce ${ }^{(2)}$, Liliana M. Pedraja-Rejas ${ }^{(3)}$ \\ Universidad de Tarapacá, (1) Centro de Ingeniería Aplicada a la Educación, (2) Instituto de Alta \\ Investigación, (3) Escuela de Ingeniería Industrial, Informática y Sistemas, Casilla 7-D, Arica-Chile. \\ (e-mail: caraneda@uta.cl; erodrigu@uta.cl; Ipedraja@uta.cl)
}

Recibido May. 29, 2013; Aceptado Jul. 7, 2013; Versión final recibida Ago. 8, 2013

\begin{abstract}
Resumen
La investigación tiene por objetivo describir la relación entre el financiamiento fiscal, la calidad del cuerpo académico y la calidad de la docencia en una población de 25 universidades chilenas. Para este fin se utilizaron fuentes de información secundaria para cada una de las variables. En el procesamiento de la información se efectuaron dos análisis de regresión lineal simple, obteniéndose los siguientes resultados: 1) El financiamiento fiscal explica en un $74 \%$ la calidad del cuerpo académico ( $p<0,01)$; 2 ); La calidad del cuerpo académico explica en un $32 \%$ la calidad de la docencia de las instituciones analizadas $(p<0,01)$. Se concluye que el financiamiento fiscal que reciben las universidades es clave para una mayor calidad de su cuerpo académico, y a su vez, un mejor cuerpo académico conlleva a lograr una mejor calidad de la docencia en las instituciones de educación superior estudiadas.
\end{abstract}

\section{Relationship Between Fiscal Funding, Quality of Faculty and University Students Retention in Chile}

\begin{abstract}
The purpose of this study was to describe the relationship between fiscal funding, quality of faculty and teaching quality. Data were collected from a population of 25 Chilean universities. The paper is based on secondary information collected from different sources. The data were analyzed employing two types of linear regression analyses. The results showed the following: 1) A significant association between fiscal funding and quality of faculty ( $<<0.01$; adjusted $R$ square 0.74$), 2)$ A significant association between quality of faculty and quality of teaching ( $p<0.01$; adjusted $R$ square 0.32 ). Finally, Chilean universities showed that fiscal funding received by them is of fundamental importance to have better quality of faculty, and in turn, high quality faculty leads to a better quality of the teaching in the higher education institutions studied.
\end{abstract}

Keywords: fiscal funding, quality of faculty, quality of teaching, higher education 


\section{INTRODUCCIÓN}

La expansión del número de estudiantes que accede a la educación postsecundaria, durante el último tiempo, ha presentado el mayor desafío para los sistemas de educación superior, donde la tradición ha sido proveer acceso gratis o altamente subsidiado de educación terciaria, lo cual en términos financieros es poco sustentable (Altbach et al., 2009), particularmente, considerando que entre los años 1970 y 2006 el número de estudiantes matriculados en instituciones de educación superior se ha incrementado de 29 a 141 millones (Freeman, 2010). La realidad en América Latina no difiere de este contexto de masificación de la educación superior, ya que durante el último tiempo se ha tomado la decisión de expandir el acceso a las instituciones de educación terciaria, lo cual ha sido entendido como una respuesta por parte del gobierno y la sociedad a la creciente demanda por educación universitaria (Maldonado-Maldonado, 2009). A este respecto, cabe señalar que las instituciones de educación postsecundaria de la región Latinoamericana muestran una gran heterogeneidad de su historia, sus cualidades y su financiamiento, sin embargo tienen en común la búsqueda de acceso democrático a la educación terciaria por medio de la expansión de su matrícula (Lebeau et al., 2012).

Junto a este creciente aumento de la matrícula de educación superior, se está dando un fenómeno paralelo de alcance internacional, el cual hace referencia a la restricción del financiamiento gubernamental de la educación superior y la privatización cada vez mayor existente en este sector. En efecto, el financiamiento del gobierno central para las universidades está declinando en muchos países del mundo (Parker, 2012). Situación que se puede considerar contradictoria, puesto que tradicionalmente se ha visualizado a la educación terciaria como un bien público que contribuye a la sociedad, mejorando el capital humano y el desarrollo económico, aunque últimamente ha comenzado a ser considerado como un bien privado (Altbach et al., 2009).

Por consiguiente, el desafío que se presenta a nivel global en términos de financiamiento de educación superior es que éste sea más sustentable. Esto, de acuerdo a Browne (2010) debido a que se observa que los recursos y los sistemas de financiamiento de la educación superior son insostenibles y necesitan de una urgente reforma. Por lo tanto, el autor propone un incremento de las contribuciones privadas y de la inversión pública más específica para apoyar la prestación de alta calidad y que el sector de educación terciaria pueda continuar creciendo para satisfacer la demanda calificada. En este sentido, el autor propone la búsqueda de mayores contribuciones de aquellos que pueden hacerlo y eliminar el subsidio general para todos los cursos, eso sí, sin perder la inversión de fondos públicos para carreras prioritarias. A esto se debe agregar además, que las políticas de educación superior en muchos países están cada vez más orientadas por la creencia de que la liberación, la facilitación y la simulación de mercados en la educación terciaria proporcionará a las instituciones académicas incentivos para mejorar la calidad de la docencia, la investigación y la productividad y estimular la innovación de los programas académicos, la investigación y los servicios para beneficiar la sociedad en su conjunto (Dill, 1997). A este respecto, existe además un creciente interés por la medida y la mejora de la eficiencia con la que funcionan las instituciones universitarias (Martín, 2008).

Por ende, dada la relevancia que tiene el financiamiento para la sustentabilidad del sistema de educación superior y por ende en el quehacer de las instituciones de educación superior, la investigación tiene como objetivo verificar si existe una relación entre el financiamiento fiscal, la calidad del cuerpo académico y la calidad de la docencia en las universidades pertenecientes al Consejo de Rectores de las Universidades Chilenas (CRUCH). Particularmente, analizar en primera instancia, el efecto que tiene el financiamiento, por medio del Aporte Fiscal Directo, sobre la calidad del cuerpo académico, es decir, en el número de académicos con doctorado y magíster contratados como jornada completa en las universidades del $\mathrm{CRUCH}$, para luego describir el impacto que tiene la calidad del cuerpo académico sobre la calidad de la docencia, es decir, sobre la retención de estudiantes, la cual hace referencia a los estudiantes que no desertan del sistema universitario, continuando sus estudios en pos de la obtención de su título profesional.

\section{MARCO TEÓRICO}

\section{Financiamiento de la educación superior}

La educación superior, desde finales de la década de los ochenta, ha venido experimentando una serie de cambios, los cuales hacen referencia principalmente a la nueva forma de interactuar entre las universidades y las autoridades gubernamentales, pues estas últimas ya no son tan receptivas a los procesos tradicionales de autorregulación que han prevalecido durante el desarrollo de las universidades por siglos. En este sentido, se observa una nueva motivación económica que está conduciendo a los Estados a redefinir la relación, presionando a las instituciones a volverse más rentables y eficientes y más productivos en el uso de los recursos públicos (Alexander, 2000). A partir de los resultados de una investigación realizada por 
Viaene y Zilcha (2011) sobre la relevancia del uso de fondos públicos para financiar la educación superior, se puede determinar que esto depende de aspectos de la economía, tales como los costos y la productividad de los sistemas de educación superior y otros parámetros que describen el proceso de formación. En efecto, la situación del financiamiento de las instituciones de educación superior se ha agudizado en el último tiempo, particularmente producto de las secuelas de la crisis financiera internacional de los años 2008 y 2009, además de la desaceleración de la economía actual, por tanto, como era de esperar, se puso una enorme presión sobre los fondos públicos destinados al sistema de educación superior (Lebeau, 2012). Con relación a esto, Sanyal y Johnstone (2011) analizaron el porcentaje del PIB que destinan los diversos países al gasto en educación superior por estudiante entre los años 1999 y 2009. Los datos muestran una disminución de este gasto en los países de América Latina y el Caribe en un $94,7 \%$. Los autores coinciden con Lebeu (2012), al considerar que la crisis económica mundial del 2008 ha tenido como consecuencia un severo impacto sobre el financiamiento de la educación terciaria.

En efecto, a pesar de un crecimiento en la apropiación de los fondos estatales de impuestos para cubrir los gastos de funcionamiento de educación superior, la inversión estatal en este sector ha disminuido considerablemente durante el último tiempo, en oposición a los cambios de la matrícula, la riqueza del Estado y el crecimiento de los presupuestos institucionales (McLendon et al., 2009). Más aún, existe evidencia de que el financiamiento por parte del gobierno central para la educación superior está declinando en muchos países. Desde Asia y Europa existe evidencia acerca de un cambio en las formas de financiación por parte de los gobiernos, quienes están conduciendo y orientando a las universidades públicas para que busquen otras fuentes alternativas de financiación y a obtener sólo una parte de los dineros públicos de acuerdo a criterios de desempeño y expandir el mercado de la educación terciara a las instituciones privadas (Parker, 2012).Por lo tanto, han sido dramáticos los cambios ocurridos en el financiamiento de la educación superior y en el debate de cómo apoyar la educación superior de masas (Altbach et al., 2009). América Latina no se encuentra exenta de esta realidad, puesto que en un documento de la UNESCO, Villanueva (2008) plantea que a partir de los años 90 los montos que se destinaban a educación debieron recortarse, ajustándose de esta forma el financiamiento de la educación superior, apareciendo en algunos sistemas el arancelamiento de los estudios y la promoción de la prestación de servicios por parte de las universidades, esto último como una forma de generar ingresos propios.

El caso de Chile no difiere de esta realidad, ya partir del año 1980 se dio inicio a una liberación del control sobre la oferta de educación superior, es decir, previo a ese año existían ocho universidades públicas con aporte fiscal, dos universidades estatales y seis privadas, de las cuales una correspondía a una universidad católica con sedes, dos católicas regionales y tres universidades seculares regionales para pasar a un sistema con diferentes niveles y con una variedad de instituciones, entre ellas ocho públicas tradicionales, catorce públicas regionales seculares, tres públicas católicas, las cuales conforman el Consejo de Rectores de Universidades Chilenas $(\mathrm{CRUCH})$. Desde ese año se dio inicio a un crecimiento de la oferta de educación superior privada. Asimismo, el peso del financiamiento de la educación superior pasó desde el Estado a los privados (Brunner, 1999). Existiendo en Chile una fuerte expansión del sector privado (Freeman, 2010).

En este nuevo contexto de la educación superior en Chile el financiamiento fiscal que se entrega a la educación terciaria está conformado por los montos de dineros distribuidos de acuerdo a lo establecido por la Ley de Presupuestos vigente, que agrupa los recursos asignados de manera directa y por medio de proyectos concursables a los que postulan las instituciones, y que se distribuyen por dos vías los recursos asignados a los estudiantes y a las instituciones. Cabe destacar que el financiamiento fiscal de la educación superior en Chile está orientado principalmente a los estudiantes, quienes obtienen el doble de los recursos que reciben las instituciones (Contraloría general de la República, 2012). De esta forma, los fondos provenientes del Aporte Fiscal Directo están disponibles sólo para las universidades del $\mathrm{CRUCH}$, beneficiando a las universidades más grandes y complejas que desarrollan investigación de manera permanente (OCDE, 2009). De este modo, el Aporte Fiscal Directo es el instrumento de financiamiento basal más relevante que el Estado destina a las universidades de Consejo de Rectores de Universidades Chilenas, el cual consiste en un subsidio de libre disponibilidad, asignados en un $95 \%$ de acuerdo a criterios históricos y un $5 \%$ restante según los indicadores de desempeño anuales vinculados con la matrícula estudiantil, el número de académicos con postgrado y el número de proyectos y publicaciones de investigación de excelencia. Por consiguiente, mediante el DFL $N^{\circ} 4$ de 1981 se establece que el Estado contribuirá al financiamiento de las universidades existentes al 31 de diciembre de 1980 y de las universidades que de ellas deriven por medio de aportes fiscales cuyo monto anual y su distribución se realizará de acuerdo a este reglamento (Divesup, 2013).

En consecuencia, la mayoría de las instituciones públicas dependen financieramente del gobierno, aunque también pueden financiarse con fondos privados a través de las matrículas, becas y contratos, así como también de la filantropía, medidas que están cada día más presentes en Estados Unidos. Asimismo, las 
universidades privadas sin fines de lucro también pueden ser dependientes del financiamiento gubernamental (Sanyal y Johnstone, 2011).

\section{Calidad del cuerpo académico}

La educación superior, como se señalaba anteriormente, enfrenta grandes desafíos y dificultades en términos de financiamiento para mejorar el desarrollo de su personal, capacitar en habilidades, mejorar su calidad, dar relevancia a sus programas y a la empleabilidad de sus graduados. Al mismo tiempo, mejorar la calidad de la educación superior y encontrar soluciones a sus mayores desafíos requiere de un fuerte involucramiento de todos los actores interesados. A este respecto, el rol y la responsabilidad del cuerpo académico resultan esenciales para que una universidad sea capaz de lograr su misión. En consecuencia, el éxito de cualquier universidad depende estrechamente de la calidad de los miembros de su cuerpo académico (Al-Ghamdi y Tight, 2013).

En este sentido, la creciente tensión entre la demanda de matrícula, los presupuestos limitados y la mayor exigencia de rendición de cuentas, han generado un ambiente desalentador para la profesión académica en todo el mundo. A esto se debe agregar el hecho de que ninguna universidad puede lograr el éxito sin un cuerpo académico bien calificado (Altbach et al., 2009). Más aún, hoy en día la calidad de los miembros del cuerpo académico se está volviendo cada vez más evidente para las personas que están fueran de la facultad, ya que la formación académica de un individuo puede ser evaluada no sólo por la lectura de sus publicaciones y documentos de trabajo, que en la actualidad se encuentran fácilmente disponibles en formato electrónico, sino que también por la creciente capacidad de criterios comparativos, tales como el número de citaciones. Asimismo, la presencia de las evaluaciones que efectúan los estudiantes de la enseñanza hace que la calidad de ésta sea mucho más fácil de evaluar desde fuera de la propia institución (Hansmann, 2012). Esta situación coincide con los resultados de un estudio cualitativo, realizado a través de entrevistas y focus group por Udam y Heidmets (2013) en Estonia, donde se establece que para los académico. Complementariamente, los resultados de la investigación efectuada por Tsinidou et al. (2010), en donde se usó el Método de Análisis Jerárquico para el procesamiento de un cuestionario, indican que la experiencia profesional, las actividades de investigación y las cualificaciones del personal académico son aspectos relevantes de la calidad académica. De hecho, un cuerpo académico de alta calidad es considerado como uno de los recursos fundamentales de excelencia de una institución de educación superior, por lo tanto un cuerpo académico de alta calidad puede considerar el mayor grado académico que posee y el prestigio de la institución de donde lo obtuvieron. A este respecto, los académicos más demandados y con mejores salarios son generalmente aquellos conocidos por su investigación y publicaciones (Astin y Antonio, 2012).

En este sentido, se puede destacar que desde fines de la década de los noventa, se han implementado una serie de medidas en Shanghai Jiao Tong University (SJTU) para mejorar la calidad del cuerpo académico. Antes de 1998, SJTU tenía un total de 1753 académicos e investigadores, de los cuales sólo un $15 \%$ tienen el grado de doctor. En consecuencia, se implementaron en la universidad una serie de programas para reclutar académicos talentosos, incluyendo iniciativas, tales como programas de catedráticos, de profesores distinguidos y de investigadores, entre otros (Wang et al., 2011). Por consiguiente, se puede establecer que existe una permanente preocupación por la calidad del cuerpo académico, debido no sólo a la relevancia que tiene para los procesos de aseguramiento de la calidad, sino que también para la productividad de las instituciones de educación superior.

\section{Calidad de la docencia}

Las universidades tienen diferentes roles en la sociedad del conocimiento, los cuales deben desarrollarse en un contexto de calidad, en el sentido de generar mecanismos que permitan asegurar la pertinencia de la entrega de los servicios académicos y resguardar el valor de los títulos y grados entregados por dichas instituciones (Rodríguez-Ponce, 2009). Por consiguiente, dada la relevancia que tiene la calidad en el quehacer de las universidades, resulta fundamental hacer referencia al concepto tradicional de calidad, el cual está asociado con la noción de entregar un producto o servicio que es distintivo y especial, que otorga además un estatus a su dueño o usuario (Green, 1994). Sin embargo, la calidad puede tener diversos significados para diferentes personas, quienes, por tanto, demandan diferentes resultados de calidad y métodos de aseguramiento (Tam, 2011). En relación a esto es posible encontrar los planteamientos de Harvey y Green (1993) quienes han desarrollado cinco dimensiones que permiten analizar la calidad en la educación superior, dimensiones que se enuncian a continuación: Calidad como excepción, es decir, considera la excelencia, por tanto, implica tener características propias que son superiores a los demás, y no alcanzables por todos, sino que sólo por los mejores; Calidad como perfección, donde se considera a un conjunto de especificaciones que deben alcanzarse para lograr la perfección; Calidad como consecución de objetivos, es decir, el grado de cumplimiento de lo que se ha propuesto; Calidad como valor por dinero, se 
refiere al retorno de la inversión; por último calidad como trasformación, o sea el paso de un estado a otro producto de un proceso. Adicionalmente, Harvey (2005) agrega los siguientes cuatro estándares: el estándar académico de logro de los estudiantes; el estándar de competencia para la práctica, los estándares de servicio proporcionados por la institución y los estándares de la organización. En consecuencia, la calidad se intersecta con estos diferentes parámetros que sirven para su análisis y medición en las instituciones de educación superior.

En efecto, es posible establecer que existe una diversidad de aspectos a considerar para evaluar la calidad. Situación que conlleva a que los procedimientos de mejoramiento de la calidad involucren necesariamente la acción de investigar por parte del personal de la institución, con la recolección frecuente y permanente de los datos y la reflexión constructiva sobre la evidencia obtenida podría dar luces sobre la calidad de la docencia y el aprendizaje y como podría ser mejorada. Los recursos que ayudan a generar la evidencia respecto a la calidad de la docencia son: los estudiantes, los graduados, el personal y la universidad (Biggs y Tang, 2011). En consecuencia, para medir la calidad de la docencia y el aprendizaje dentro de las instituciones de educación superior se deben involucrar diversos indicadores que sean significativos en información individual y del funcionamiento de la institución. Por lo tanto, un indicador como la tasa de retención es bastante útil desde una perspectiva social y económica, ya que logra simplificar la complejidad de la experiencia de la educación superior (Chalmers, 2008).

Por lo tanto, de acuerdo a lo señalado previamente, se desprenden las siguientes hipótesis del estudio:

H-1 El financiamiento fiscal de las instituciones de educación superior impacta positivamente sobre la calidad del cuerpo docente.

H-2 La calidad del cuerpo docente de las instituciones de educación superior impacta positivamente sobre la retención de estudiantes universitarios en Chile.

La siguiente figura muestra de manera integrada el conjunto de hipótesis de la investigación:

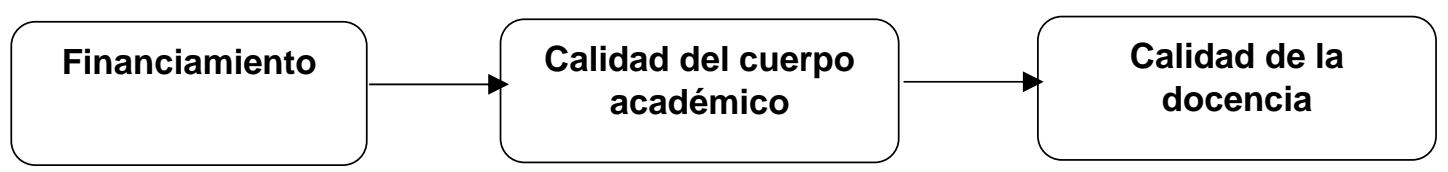

Fig. 1: Modelo de la investigación

\section{METODOLOGÍA}

La metodología empleada es de orden cuantitativa, asimismo el propósito de la investigación es exploratorio y descriptivo. Cabe señalar que se trabajó con información obtenida de la revisión de datos secundarios. A este respecto, la muestra estuvo compuesta por las 25 universidades que forman parte del Consejo de Rectores de Universidades Chilenas (CRUCH). Cabe consignar que la técnica de muestreo fue intencionada, puesto que se trabajó con las instituciones que recibieron durante el año 2011 Aporte Fiscal Directo por parte del Estado, siendo ese el criterio de selección. Por lo tanto, el estudio no es representativo y además es no probabilístico.

Como fuente de información para cada una de las variables se utilizó el Anuario Estadístico 2011 del $\mathrm{CRUCH}$, el documento de Financiamiento Fiscal a la Educación Superior 2011, elaborado por la Contraloría General de la República (2012) y una presentación realizada en mayo de 2012 por parte del Ministerio de educación de Chile. Las variables del estudio fueron las siguientes: para medir el financiamiento de las instituciones de educación superior el indicador usado fue el Aporte Fiscal Directo que recibieron dichas instituciones en el año 2011, de acuerdo al informe Financiamiento Fiscal a la Educación Superior 2011, de la Contraloría General de la República (2012).

Para medir la calidad del cuerpo académico se usaron los siguientes indicadores: el número de doctores contratados en jornada completa y el número de académicos con el grado de magíster contratado en jornada completa en cada universidad. Cabe precisar que para ambos indicadores se calculó el Alpha de Cronbach, el cual arrojó como valor 0,847, evidenciando una alta fiabilidad. Debido a que el número de 
doctores contratados en jornada completa y el número de académicos con el grado de magíster contratado en jornada completa en cada universidad se encuentran altamente correlacionadas entre sí, al hacer referencia a la calidad de los académicos en las universidades se construye una variable sumando los totales de ambas, construyendo de esta forma la variable "Calidad del cuerpo académico".

Finalmente, para medir la calidad de la docencia se utilizó el porcentaje de retención al tercer año (2011) en las universidades estudiadas. La figura 2 muestra la operacionalización del modelo conceptual de la investigación.

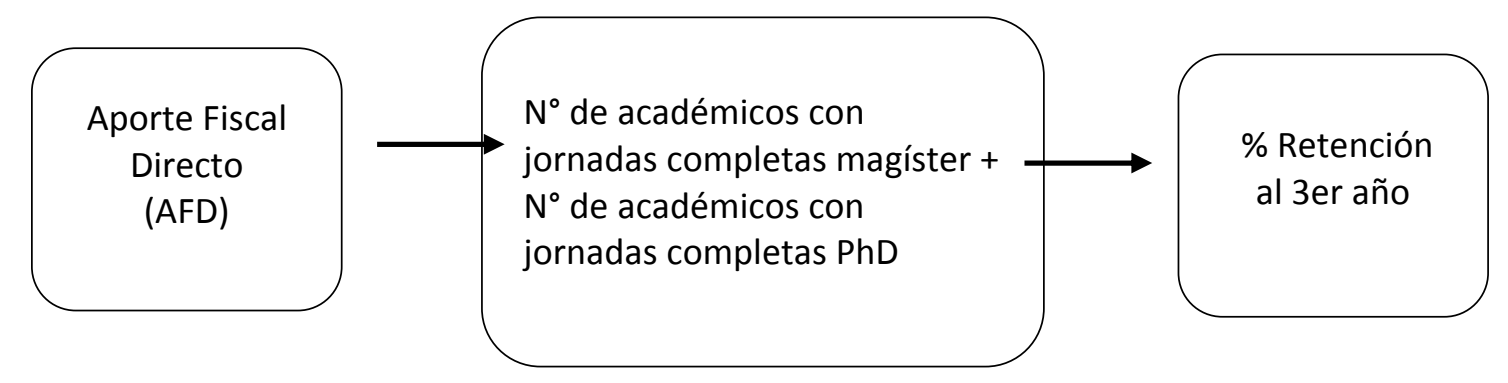

Fig. 2: Relación de las variables de la investigación

Como técnica de análisis se utilizó la regresión lineal simple, con las siguientes ecuaciones de regresión:

$V x=\beta 0+a \beta 1+\varepsilon i$

Donde, $V x$ es la variable dependiente: calidad del cuerpo académico; $\beta 0$ : constante del modelo, a la variable independiente, financiamiento, es decir el Aporte Fiscal Directo que reciben las universidades del $\mathrm{CRUCH}$; $\beta 1$ es el factor de ponderación y $\varepsilon i$ : error residual del modelo.

Del mismo modo para explicar el impacto de la calidad del cuerpo académico sobre la calidad de la docencia se ocupa la siguiente ecuación de regresión:

$V x=\beta 0+a \beta 1+\varepsilon i$

Donde, Vx es la variable dependiente: calidad de la docencia, es decir, porcentaje de retención alumnos de tercer año (2011); $\beta 0$ : constante del modelo, a la variable independiente, calidad del cuerpo académico académica; $\beta 1$ es el factor de ponderación y zi: error residual del modelo.

\section{RESULTADOS}

En los resultados se puede observar la relación entre la variable independiente financiamiento fiscal, medido a través del Aporte Fiscal Directo y la variable dependiente, calidad del cuerpo académico, es significativa $(p<0,01)$, tal como se puede observar en la tabla 1.

Tabla 1. Resumen modelo de regresión: Financiamiento y calidad del cuerpo académico

\begin{tabular}{lc}
\hline & Calidad del cuerpo académico \\
\hline$R$ & $0,868^{*}$ \\
$R$ cuadrado ajustado & 0,743 \\
Test t constante & 1,451 \\
Coeficiente B no estandarizado constante & 101,740 \\
Coeficiente B no estandarizado AFD & $6,329 \mathrm{E}-005$ \\
Test T & 8,382 \\
Significancia test T & 0,000 \\
\hline Fuente: Procesamiento del cuestionario en SPSS & \\
Variable predictora: Financiamiento (Aporte Fiscal & \\
Directo) & \\
*Significancia al 1\% & \\
\hline
\end{tabular}


A partir de los resultados que se puede establecer que el financiamiento por Aporte Fiscal Directo impacta de manera directa y fuerte $(R \quad 0,868)$ en la calidad del cuerpo académico, teniendo una capacidad explicativa del financiamiento de un $74,3 \%$. Asimismo su significancia estadística es bastante alta $($ Test $t=$ $8,382 ; p<0,01)$.

Por consiguiente, es posible señalar, a través del análisis de regresión efectuado, que el financiamiento, mediante el Aporte Fiscal Directo tiene un impacto significativo y directo sobre la calidad del cuerpo académico, por ende, sobre la base de los datos observados no hay evidencias para rechazar la hipótesis 1.

Con relación al impacto que tiene la calidad del cuerpo docente sobre la calidad de la docencia, medida esta última a través de la retención de los alumnos de tercer año de las universidades estudiadas, se puede establecer que la relación es significativa y directa, como se puede apreciar en la tabla 2.

Tabla 2. Resumen modelo de regresión: Calidad del cuerpo académico y calidad de la docencia

\begin{tabular}{lc}
\hline & Calidad de la docencia \\
\hline$R$ & $0,590^{*}$ \\
R cuadrado ajustado & 0,320 \\
Test t constante & 28,257 \\
Coeficiente B no estandarizado constante & 57,886 \\
Coeficiente B no estandarizado AFD & 0,010 \\
Test T & 3,507 \\
Significancia test T & 0,002 \\
\hline
\end{tabular}

Fuente: Procesamiento del cuestionario en SPSS

Variable predictora: Calidad del cuerpo académico

${ }^{*}$ Significancia al $1 \%$

Por consiguiente se puede determinar que la calidad del cuerpo académico tiene un impacto significativo sobre la calidad de la docencia. Es importante precisar que existen otras variables omitidas en el modelo, como lo indica el test $\mathrm{T}$ de la constante. A pesar de eso resulta interesante saber que la calidad del cuerpo académico medida por el número de académicos con doctorado y el número de académicos con magíster logra explicar el $32 \%$ de la varianza de la calidad de la docencia. Es decir, en este caso como el porcentaje de alumnos que no deserta del sistema de educación superior es un fenómeno que va más allá de los atributos individuales de los alumnos, como lo plantea Georg (2009), quien indica que no se ha dado la suficiente relevancia a los aspectos institucionales de las universidades, como lo son la regulación del programa de estudio, la calidad de la enseñanza y de orientación, factores que contribuyen a reducir la deserción universitaria. En consecuencia, la retención, como indicador de la calidad de la docencia, hace referencia a la contracara de la deserción. Sus estadísticas entregan información relevante respecto a cuantos alumnos continúan sus estudios y consiguen avanzar hacia la obtención de su título profesional (Centro de Estudios Mineduc, 2012).

En efecto, sobre la base de los resultados no hay evidencia para rechazar la hipótesis número 2 planteada, evidenciando la relevancia que tiene para la calidad de la docencia, medida como el porcentaje de retención de los alumnos de tercer año de las universidades estudiadas. Por lo tanto, los resultados alcanzados refuerzan la idea de Al-Ghamdi y Tight, (2013) quienes indican que las universidades esperan que los miembros del cuerpo docente mejoren la calidad de la docencia. De esta forma, dada su trascendencia, la enseñanza de alta calidad debe ser entregada por el cuerpo académico altamente calificado y comprometido con su permanente desarrollo profesional (Gibbs, 2010). Esto se vincula a la tendencia actual de que las universidades están principalmente preocupadas por la calidad de la docencia y la investigación. A este respecto se puede mencionar lo sucedido en Argentina en 1995, donde el Fondo para el Mejoramiento de la Calidad apoyó al mejoramiento de la calidad de los docentes por medio del otorgamiento de becas de postgrado, ya que en ese entonces menos del $6 \%$ del plantel docente tenía un título de postgrado (Samoilovich, 2008).

Asimismo, otro de los aspectos relevantes encontrados en los resultados de la investigación muestran, tal como la literatura lo indica, que la calidad de la educación superior depende de la concentración de financiamiento y de la investigación (Gidley, 2010). A este respecto un estudio realizado por Beath et al. (2012) buscó comprender cómo una cultura del sistema universitario depende de manera sistemática de la forma de financiamiento, donde la cultura capta el énfasis puesto en la investigación y la docencia. Los 
investigadores construyeron un modelo en el que la restricción presupuestaria que enfrenta el sector de la educación superior desempeña un papel fundamental en la determinación del tipo de cultura de investigación y de docencia que se generará. Con este fin se utilizó un tipo genérico del modelo de financiación y se pudo descubrir el surgimiento de dos fenómenos culturales, tales como "elites de investigación" y la "división binaria". La división binaria hace referencia a la diferenciación entre instituciones politécnicas y las universidades en el Reino Unido, donde sólo en las últimas se podría entregar el grado de investigación. Por otro lado, por elites de investigación se entiende a los grupos de universidades donde se pone énfasis en la investigación. En consecuencia, se puede establecer, y de acuerdo a la literatura revisada y los resultados obtenidos por la investigación, que el financiamiento de la educación superior es un factor determinante en la calidad de su cuerpo académico y el logro de la calidad de la docencia, afectando directamente en el quehacer de la universidad.

La calidad de la docencia en la educación superior en el Reino Unido, por ejemplo, tiene en particular su énfasis en la atención personal a los estudiantes y en el logro de alto nivel de los resultados del aprendizaje, situación que se ha sostenido a pesar de la fuerte presión sobre los recursos (Ramsden, 2008). En efecto, esta asociación entre calidad y financiamiento no es arbitraria, pues, de acuerdo a los planteamientos de Bleiklie (2011) existe una tendencia a concentrar la financiación en las mejores instituciones, situación que ha sido fomentada por los propios gobiernos que se ven a sí mismos operando en condiciones de financiación restringida y con la necesidad simultánea de generar instituciones de excelencia con el fin de hacer más competitiva la economía del país.

\section{CONCLUSIONES}

Abordar el financiamiento fiscal dentro de la educación superior no resulta un tema sencillo de analizar, debido a la gran cantidad de factores asociados que lo pueden llegar a determinar.

El financiamiento en las instituciones de educación superior se configura como un elemento vital y catalizador para el funcionamiento de las universidades, más aún, se puede establecer que el financiamiento es crucial para la supervivencia de dichas instituciones, y clave al momento de hablar de la calidad del quehacer académico y de su cuerpo docente, aspectos que, tal como lo demuestran los resultados alcanzados, tienen un impacto directo sobre los estudiantes.

Este tema cobra relevancia hoy en día, donde prima la masificación de la educación superior y la reducción de los recursos por parte del Estado, situación que traspasa fronteras, y es uno de los desafíos fundamentales que deben enfrentar las instituciones universitarias.

En consecuencia, de acuerdo a los resultados de la presente investigación, los recursos financieros impactan de manera directa sobre la calidad del cuerpo académico, por consiguiente, instituciones con mayores recursos económicos, cuentan con un cuerpo docente de mayor calidad y con mejores cualificaciones, es decir, con mayor calificación académica, evidenciado esto en sus grados de magíster y doctorado. A su vez, un mejor cuerpo académico, conlleva a una mejor calidad de la docencia, lo cual impacta positivamente en la retención de los estudiantes, logrando, en mayor medida, que éstos puedan concluir de manera exitosa sus planes de estudios y obtener así su título profesional.

\section{REFERENCIAS}

Alexander, K., The changing face of accountability: Monitoring and assessing institutional performance in higher education, Journal of Higher Education, 71(4), 411-431 (2000).

Al-Ghamdi, S. y M. Tight, Selecting and Developing High-Quality Academic Staff, Higher Education Dynamics, 30, 83-93 (2013).

Altbach, P., L. Reisberg y L. Rumbley, Trends in global higher education: Tracking an academic revolution, UNESCO Pub. Francia (2009).

Astin, A., y A. Antonio, Assessment for excellence: The philosophy and practice of assessment and evaluation in higher education, $2^{\text {a }}$ edición, Rowman \& Littlefield, USA (2012).

Beath, J., J. Poyago-Theotoky, y D. Ulph, University funding systems: impact on research and teaching, Economics: The Open-Access, Open-Assessment E-Journal, 6(2), 1-23 (2012).

Biggs, J., Tang, C., Teaching for quality learning at university, $4^{\text {a }}$ edición, McGraw-Hill, UK (2011). 
Bleiklie, I., Excellence, Quality And The Diversity Of Higher Education Systems. En Questioning Excellence in Higher Education. Questioning Excellence in Higher Education, 3, 21-35 (2011).

Browne, J., Securing a sustainable future for higher education: an independent review of higher education funding and student finance, Department for Business, Innovation and Skills, UK (2010).

Brunner, J. J., La educación superior en Chile: tendencias y perspectivas. En Conferencia Organizada por el Ministerio de Educación y el Banco Mundial, Montevideo (1999).

Centro de Estudios Mineduc, Deserción en la educación superior en Chile, Serie Evidencias, 1(9), 1-12 (2012).

Contraloría General de la República, Financiamiento Fiscal a la Educación Superior 2011. Contraloría General de la República División de Análisis Contable Área Empresas Públicas y Universidades, (2012)

Chalmers, D., Teaching and learning quality indicators in Australian universities. Outcomes of higher education: Quality relevance and impact, Programme on Institutional Management in Higher Education, Australia, 8-9 de septiembre (2008).

CRUCH, Anuario estadístico 2011, Santiago de Chile (2012).

Dill, D., Higher education markets and public policy, Higher Education Policy, 10(3-4), 3-4 (1997).

DIVESUP, Aporte Fiscal Directo.

http://www.divesup.cl/index2.php?id_portal=38\&id_seccion=3063\&id_contenido=12222 Fecha de Acceso: 03 de abril (2013).

Freeman, R., What Does Global Expansion of Higher Education Mean for the United States?, In American Universities in a global market, by C. Clotfelter, pp. 373-404 University of Chicago Press, USA (2010).

Georg, W., Individual and institutional factors in the tendency to drop out of higher education: a multilevel analysis using data from the Konstanz Student Survey, Studies in Higher Education, 34(6), 647-661 (2009).

Gibbs, G., Dimensions of quality, Higher Education Academy, York (2010).

Gidley, J., G., Hampson, L., Wheeler, y E., Bereded-Samuel, From access to success: An integrated approach to quality higher education informed by social inclusion theory and practice, Higher Education Policy, 23(1), 123-147 (2010).

Green, D., What Is Quality in Higher Education? Taylor \& Francis, Bristol, PA (1994).

Hansmann, H., The Evolving Economic Structure of Higher Education, The University of Chicago Law Review, 79(1),159-183 (2012).

Harvey, L., A history and critique of quality evaluation in the UK, Quality Assurance in Education, 13(4) 263276 (2005).

Harvey, L., y D. Green, Defining quality, Assessment \& Evaluation in Higher Education, 18(1) 9-34 (1993).

Lebeau, Y., R. Stumpf, R. Brown, M. Lucchesi, y M. Kwiek, Who shall pay for the public good? Comparative trends in the funding crisis of public higher education, Compare: A Journal of Comparative and International Education, 42(1), 137-157 (2012).

Maldonado-Maldonado, A. Latin American higher education: Hope in the struggle? In Leadership for worldclass universities: Challenges for developing countries by P. G. Altbach, pp. 73-93: Routledge, New York (2009).

Martín, R. La medición de la eficiencia universitaria: una aplicación del Análisis Envolvente de Datos, Formación Universitaria, 1(2), 17-26 (2008).

Mclendon, M., J. Hearn, y C. Mokher, Partisans, professionals, and power: The role of political factors in state higher education funding, The Journal of Higher Education, 80(6), 686-713 (2009). 
MINEDUC, Presentación en Consejo de Rectores de las Universidades Chilenas, 26 de abril de 2012 (2012).

OCDE. La educación superior en Chile. Revisión de políticas nacionales de educación. OCDE y el Banco Internacional para la Reconstrucción y el Desarrollo, (2009).

Parker, L. D. From privatised to hybrid corporatised higher education: a global financial management discourse, Financial Accountability \& Management, 28(3), 247-268 (2012).

Ramsden, P. The future of higher education teaching and the student experience. The Higher Education Academy, 30, 1-21 (2008).

Rodríguez-Ponce, E., El rol de las universidades en la sociedad del conocimiento y en la era de la globalización: Evidencia desde Chile, Interciencia, 34(11), 822-829 (2009).

Samoilovich, D. Senderos de innovación. Repensando el gobierno de las universidades públicas en América Latina. InTendencias de la educación superior en América Latina y el Caribe, por Gazzola, A., y Didriksson, A. (Ed.). pp. 319-380, IESALC, UNESCO (2008).

Sanyal, B. y Johnstone, B. International trends in the public and private financing of higher education, Prospects, 41(1), 157-175 (2011).

Tam, M., Measuring Quality and Performance in Higher Education, Quality in Higher Education, 7(1), 47-54 (2001).

Tsinidou, M., V. Gerogiannis, y P. Fitsilis. Evaluation of the factors that determine quality in higher education: an empirical study, Quality Assurance in Education, 18(3), 227-244 (2010).

Udam, M., y M. Heidmets, Conflicting views on quality: interpretations of 'a good university'by representatives of the state, the market and academia. Quality in Higher Education, (ahead-of-print), 1-15, (2013).

Viaene, J. y I. Zilcha, Public funding of higher education. No. 3606. CESifo working paper: Economics of Education, (2011).

Villanueva, E. Reformas de la educación superior: 125 propuestas para la educación superior en América Latina y El Caribe. En: Tendencias de la educación superior en América Latina y el Caribe. By A. Gazzola, y A. Didriksson, (Ed.) pp. 241-296 IESALC, UNESCO (2008).

Wang, Q., Q. Wang, y N. Liu, Building World-Class Universities in China: Shanghai Jiao Tong University, In The road to academic excellence: The making of world-class research universities, by P. Altbach y J. Salmi, 33-62, World Bank Publications, USA (2011). 